\title{
Clinical Outcomes and Finite Element Method Results of Anterior Cervical Discectomy and Fusion Using H-Beam Shaped Allospacer: A Comparison with Rim-Shaped Allospacer
}

\author{
Kyoung Jae Park ${ }^{1}$, Dae Hwan Kim ${ }^{2}$, Kyoung Duck Park ${ }^{1}$, Jun Hee Park ${ }^{1}$, Nam Kyu Yoo ${ }^{1}$, Ki Hong Cho ${ }^{1}$, \\ Sang Hyun Kim ${ }^{1}$ \\ Departments of ${ }^{1}$ Neurosurgery, ${ }^{2}$ Physical Medicine and Rehabilitation, Ajou University School of Medicine, Suwon, \\ Republic of Korea
}

Corresponding author:

Sang Hyun Kim

Department of Neurosurgery, Ajou University School of Medicine, 164, World cup-ro, Yeongtong-gu,

Suwon 16499, Republic of Korea

Tel: $+82-31-219-5230$

Fax: +82-31-219-5238

E-mail: kaliemd@naver.com

Received: August 5, 2019

Revised: September 7, 2019

Accepted: September 17, 2019

\begin{abstract}
Objective: The main purpose of this study was to evaluate radiologic outcomes and Finite element method (FEM) results of anterior cervical discectomy and fusion (ACDF) using $\mathrm{H}$-beam shaped allospacer, in comparison with rim-shaped allospacer. Methods: From March 2011 to February 2014, 95 patients underwent ACDF using allospacers (H-beam shaped and rim shaped). Sixty-two patients were divided into 2 groups: $\mathrm{H}$ - beam shaped allospacer in group $\mathrm{A}(\mathrm{n}=31)$; rim shaped allospacer in group $B(n=31)$. We retrospectively estimated clinical outcomes, such as, neck and arm pain, radiographic fusion rate and adverse effects. In the FEM study, we compared the performance of 3 different shaped allospacer; i.e., $\mathrm{H}$-beam shaped, $\mathrm{H}$-hole shaped, rim-shaped. We tested the effectiveness of stress distribution from the 3 types of allospacer, using the evaluation criteria of (a) compression; (b) shear; and (c) torsion, under the same load. Results: Neck and arm pain was reduced similarly by $63 \%$ to $73 \%$ in 2 groups. Fusion rates after 1 year in group A and B were $100 \%$ and $98 \%$ retrospectively. The complication rates of breakage and displacement in Group B were 16\% and 3\%. Group A show no adverse effects. From the FEM study, in the cases of the (a), (b), (c), we found that H-beam shaped allospacer resulted in effectiveness stress distribution and diversification regarding compression, shear and torsion compared with rim shaped allospacer. Conclusion: In ACDF using allospacer, $\mathrm{H}$-beam shaped allospacer shows more stable fusion rates with low incidence of complication compared with rim shaped allospacer.
\end{abstract}

Key Words: Cervical vertebrae; Finite element analysis; Spinal fusion

\section{INTRODUCTION}

Anterior cervical discectomy and fusion (ACDF) is a standard neurosurgical procedure used in treatment of cervical spondylotic radiculopathy ${ }^{2,4,9,12,16,19,20,22)}$. To supplement the bone grafts, various methods of anterior cervical interbody fusion have been developed over the past 4 decades; i.e., stand alone ${ }^{12}$, autograft and plate-screw fixation ${ }^{18)}$, cage and plate-screw fixation $^{7)}$, allograft and plate-screw fixation ${ }^{23)}$.

Using autograft bone or other alternatives has shown various complication -breakage, displacement, fusion failure, donor site complication ${ }^{6,8,11,14)}$. Especially using iliac crest autograft bone, donor site complication was associated with significant shortand long-term morbidity such as infection, pelvic fracture, meral- gia paresthetica, wound hematoma, loss of sensation and especially postoperative pain in up to about $20 \% 3,10,17,21$ )

Many journals have been reported that among various methods of anterior cervical interbody fusion, allobone graft and plate-screw fixation is as effective as other methods in terms of bone fusion rate, graft stability- displacement, subsidence, implant failure ${ }^{5,14,18,23)}$.

But, of the same allobone graft and plate-screw fixation methods, journals on clinical outcomes compared in accordance with the shape of allobone spacers are rare. In this paper, we compare the clinical outcomes relating shape of allobone spacer. Also, to compare the implant stability, finite element method (FEM) was performed in accordance with the 3 allobone spacer shape. 


\section{MATERIALS AND METHODS}

\section{Patients}

Between March 2011 and February 2014, 62 patients were enrolled among the total 95 patients treated with multi-level segmental anterior cervical fusion in our institute were retrospectively reviewed. Inclusion criteria include cervical disc disease, spondylolisthesis and segmental instability, no improvement after conservative treatment for at least 6 weeks, follow-up period for more than 12 months and 62 patients were included. Thirty-three patients who had infection, neoplastic disease, hemorrhagic disease, metabolic bone disease, ossification of the posterior longitudinal ligament, combined with posterior operation and follow-up period for less than 12 months were excluded. Enrolled 62 patients were divided into 2 groups. These 31 patients (Group A) underwent ACDF using H-beam shaped allobone cervical spacer (CGBIO, Seoul, Korea) and 31 patients (Group B) underwent rim shaped allobone cervical spacer (KBB, Seoul, Korea) combined with rigid plate and screw system.

All patients were treated using a standard Robinson and Smith's anterior cervical approach by one neurosurgeon. After the completion of discectomy and any necessary decompression under microscope, the cartilaginous endplates were removed. Endplates were cleaned and fashioned with curettes and a high-speed pneumatic drill, and we tried to preserve the endplate as much as possible to prevent cage subsidence. After intervertebral space was widened sufficiently using Caspar screw, an appropriate-size allospacer packed with demineralized bone matrix (Integra, Carlsbad, CA, USA) was inserted without impact on the spacer itself to avoid the breakage or crack. And then a rigid anterior cervical spine plate (DePuySynthes, Warsaw, IN, USA) was applied in all cases. After removing Caspar screw, anterior plate was fixed by the method that fixing screws to upper and lower level were achieved upward and downward respectively to oblique direction of the vertebral bodies. Before closing the superficial layers, a lateral radiograph was obtained, and the correct position of the implant and overdistraction was checked by comparison with the preoperative image. All patients were treated with the same protocol after surgery and consisting of immobilization with the Philadelphia cervical collar for 3 to 4 weeks.

All patients were followed-up via serial cervical plain radiographs (serial anteroposterior and lateral image) and observation of clinical outcomes 3, 6, and 12 months postoperatively. Lateral flexion-extension radiography and computed tomography scan was performed for all patients to 12 months after surgery to evaluate bone fusion. Bone fusion was deemed to have occurred if visualization of trabecular bridging across the bone-graft interface, absence of radiolucent gaps between the endplate and graft, restriction of motion $(0-2 \mathrm{~mm})$ of the fusedlevel spinous process on flexion-extension.

A definition of subsidence in terms of spinal biomechanics is presented in the paper. Subsidence is defined as sinking of a body with a higher elasticity modulus (e.g., graft, cage, spacer) in a body characterized by a lower elasticity modulus (e.g., vertebral body), resulting in 3 dimensional changes of the spinal geometry ${ }^{13)}$. Subsidence was defined as a decrease of $3 \mathrm{~mm}$ or more of anterior or posterior disc height from that measured on the immediate postoperative radiograph. Any excessive subsidence decreases the interbody space and produces both segmental and general kyphotic change of the spine. This may cause destabilization of the screw-plate and/or screw-bone interfaces (e.g., pulling-out, altered angulation or breakage of the screws).

A definition of spacer breakage was defined as definite recognition of linear fracture or fracture particles of allospacer on postoperative radiograph during postoperative follow-up period.

We estimated clinical outcomes such as neck and arm pain (visual analogue scale [VAS] score), radiographic fusion rate, breakage and displacement. Statistical significance was used for the Fisher's exact test.

\section{Statistical Analysis}

In the FEM study, we compared the implant stability of 3 different shaped allobone cervical spacer; i.e., H-beam shaped, H-hole shaped, rim-shaped (Fig. 1). We tested the
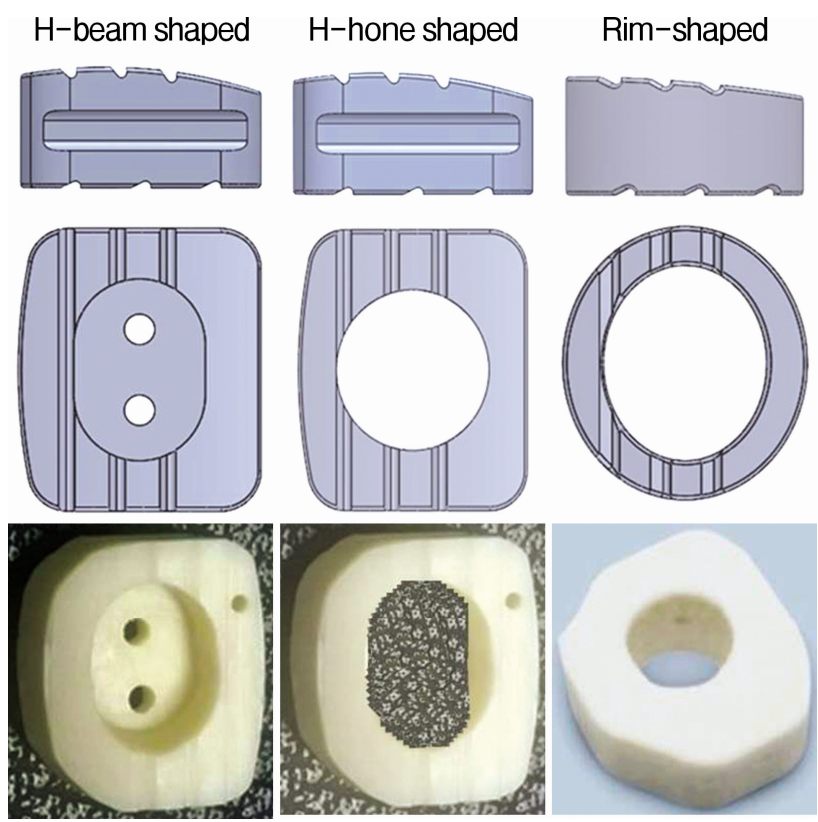

Fig. 1. In Finite element method study, schematic pictures in three different shaped allobone cervical spacer; e.g., H-beam shaped, $\mathrm{H}$ hole shaped, and rim-shaped. 
effectiveness of stress distribution from the 3 types of allospacer, using the evaluation criteria of (a) compression; (b) shear; and (c) torsion, under the same load. We implemented FEM study environment according to the standards of American Society for Testing and Materials (ASTM) F-2077. In FEM study, compression, shear, torsion was set to load condition of each $2,000 \mathrm{~N}, 3 \mathrm{Nm}, 2,000 \mathrm{~N}$. Under the same load, the simulation was conducted using a jig of stainless steel material in the upper and lower implant.

\section{RESULTS}

Thirty-one patients were enrolled in Group A and underwent ACDF using H-beam shaped allobone spacer; 10 patients had a 1-level fusion, 19 patients had a 2-level fusion and 2 patients had a 3-level fusion. Thirty-one patients were enrolled in Group $\mathrm{B}$ and underwent ACDF using rim shaped allobone spacer; 18 the patients had a 1-level fusion, 7 patients had a 2-level fusion 6 patients had a 3-level fusion (Table 1). The mean age in each group were 54.4 \pm 13.1 years in Group A and 52.6 \pm 11.7 years in Group B. There were no statistically significant diffe- rences in age, sex ratio, follow-up period, and inclusion criteria between the groups. Diabetes mellitus and T-score of bone mineral density (BMD) were investigated among factors that could affect implant failure, such as breakage or displacement. Those who had a history of diabetes mellitus numbered 8 in group A and 10 in group B, while the mean value of BMD was -1.6 and -2.0 , respectively. However, neither showed significant differences between the 2 groups. There was statistically significant difference in surgical levels. Single level ACDF surgeries were frequently performed in Group B and multi-level ACDF surgeries (more than 2 levels) were more frequently performed in Group A. The fusion rates in the 12 months were 100\% in Group A, and 96.8\% Group B (Table 1). There was no significant difference in fusion rates between Group $\mathrm{A}$ and $\mathrm{B}$ (Fisher's exact test, $\mathrm{p}>0.05$ ) (Table 1).

We compared the complication, such as breakage, displacement of Group A and Group B. The overall breakage rates in Group A and Group B were 0\%(0/31) and 16.1\%(5/31), retrospectively. The incidences of defined subsidence in Group A and Group B were 0\% (0/31) and 16.1\% (5/31), retrospectively which were exactly same as those of breakage (Table 2 ). The breakage rate among patients who experienced in H-beam

Table 1. Demographic data and surgical levels of the patients in group A and B

\begin{tabular}{|c|c|c|c|}
\hline & Group A (H-beam shaped) & "Group B (rim-shaped) & p-value \\
\hline No. of cases & 31 & 31 & \\
\hline Degenerative disc disease & 25 & 22 & 0.483 \\
\hline Trauma & 6 & 9 & 0.477 \\
\hline Age (year) & $54.4 \pm 13.1$ & $52.6 \pm 11.7$ & 0.501 \\
\hline Sex & & & 0.125 \\
\hline Male & 20 & 22 & \\
\hline Female & 11 & 9 & \\
\hline DM & 8 & 10 & 0.093 \\
\hline $\mathrm{BMD}$ & $-1.6 \pm 0.8$ & $-2.0 \pm 1.1$ & 0.802 \\
\hline Follow-up period & 20 & 18 & 0.746 \\
\hline \multicolumn{4}{|l|}{ Surgical level } \\
\hline 1 level & 10 & 18 & $0.037^{*}$ \\
\hline 2 level & 19 & 7 & $0.031^{*}$ \\
\hline 3 level & 2 & 6 & $0.048^{*}$ \\
\hline Fusion rate (Post-op 12 months) & 31 (100.0\%) & $30(96.8 \%)$ & 0.502 \\
\hline
\end{tabular}

Post-op: postoperative; DM: diabetes mellitus; BMD: bone mineral density.

'Statistically significant.

Table 2. Breakage and displacement of allospacer in group $A$ and $B$

\begin{tabular}{|c|c|c|c|c|c|c|c|c|c|}
\hline & \multicolumn{4}{|c|}{ Group A $(n=31)$} & \multicolumn{4}{|c|}{ Group B $(n=31)$} & \multirow[b]{2}{*}{ p-value } \\
\hline & $\begin{array}{l}1 \text { level } \\
(n=10)\end{array}$ & $\begin{array}{c}2 \text { levels } \\
(n=9)\end{array}$ & $\begin{array}{c}3 \text { levels } \\
(n=2)\end{array}$ & Total & $\begin{array}{l}1 \text { level } \\
(n=18)\end{array}$ & $\begin{array}{c}2 \text { levels } \\
(n=7)\end{array}$ & $\begin{array}{c}3 \text { levels } \\
(n=6)\end{array}$ & Total & \\
\hline Breakage & 0 & 0 & 0 & $0(0.0 \%)$ & 2 & 1 & 2 & $5(16.1 \%)$ & $0.026^{*}$ \\
\hline Displacement & 0 & 0 & 0 & $0(0.0 \%)$ & 1 & 0 & 0 & 1 (3.2\%) & 0.5 \\
\hline
\end{tabular}

Statistically significant $(p<0.05)$. 
shaped spacer was significantly less likely than that using rimshaped spacer (Fisher's exact test, $\mathrm{p}<0.05$ ). There was no particular problem when using the H-beam shaded spacer, but we could see that breakage occurs when using the rim-shaped spacer. The bone fusion between the upper and lower vertebrae was good, and the plate and screw looked stable in Group A patient. Bone fusion was relatively good in Group B, but the plate and screw looked unstable (Fig. 2). The overall displacement rates in Group A and Group B were $0 \%(0 / 31)$ and $3.2 \%(1 / 31)$, respectively. The displacement rate among patients who experienced in H-beam shaped spacer was not significantly less likely than that using rim-shaped spacer (Fisher's exact test, $\mathrm{p}>0.05$ ) (Table 2).

We investigated the VAS for the preoperation and postoperation neck pain and arm pain. Neck pain was reduced in Group A and Group B by $68 \%$ and $73 \%$, respectively. Also, arm pain was reduced in Group A and Group B by 63\% and $72 \%$ respectively. In the VAS score, Group B is a slightly better result than the Group A (Table 3).

In FEM study, compression was set to load condition of each 2,000 $\mathrm{N}$ in the simulation. Under the same load, peak von mises stress (PVMS) values were checked to each 90.8 $\mathrm{MPa}, 126.3 \mathrm{MPa}, 365.1 \mathrm{MPa}$ in H-beam, H-hole, rim shaped allobone spacer. In the shear condition setting to $3 \mathrm{Nm}$, PVMS values was measured to each $61.2 \mathrm{MPa}, 85.0 \mathrm{MPa}, 621.2 \mathrm{MPa}$ in 3 shaped allobone spacer. In the torsion condition setting to $2,000 \mathrm{~N}$, PVMS values was measured to each $244.7 \mathrm{MPa}$, 261.9 MPa, 863.7 MPa in 3 different shaped spacers (Fig. 3).

\section{DISCUSSION}

As many journals have been published, in cervical interbody grafts, allograft has the effectiveness as well as other material $^{5,14,15,18,23)}$. Also, allograft is currently used in the clinic of the authors. But as it mentioned in many journals, allobone cervical spacer intermittently showed complications such as breakage, displacement also in our hospital. In this study, to reduce these complications, we compared the clinical outcomes relating shape of allobone spacer (H-beam shaped or rim shaped).

First, numerous studies have concluded that ACDF with allograft derives clinically satisfactory results in patients with cervical radiculopathy and myelopathy ${ }^{1,6)}$. The results of this study ensure this conclusion. As seen in postoperative VAS score, H-beam shaped allograft with ACDF results in substantial pain relief, improvements in quality of life likewise rim-shaped allograft with ACDF.

Second, the displacement rate among patients who experienced in H-beam shaped spacer was not significantly less likely
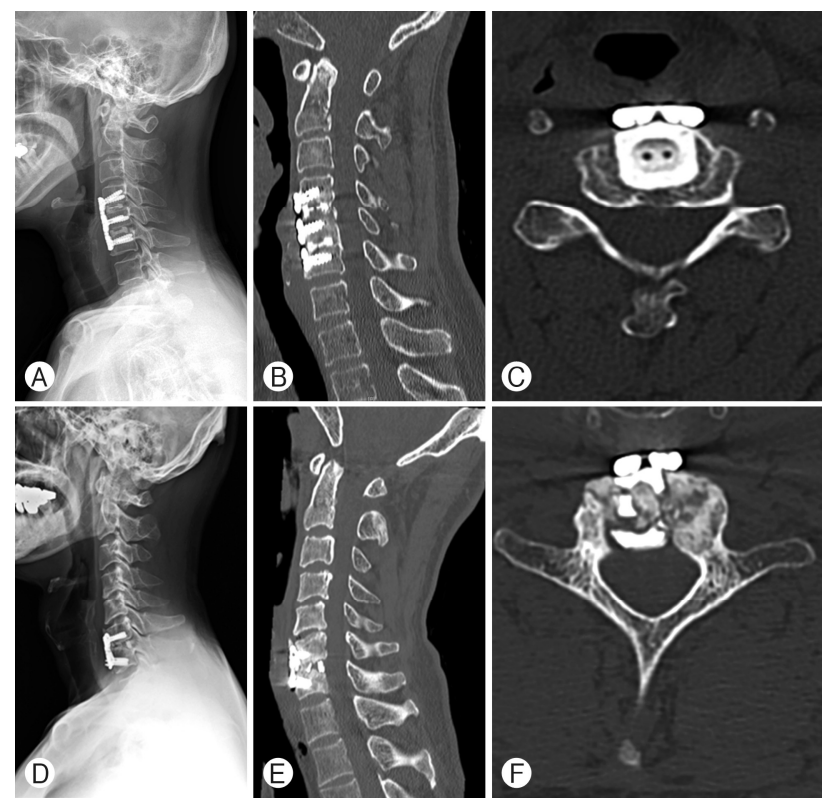

Fig. 2. Comparison c-spine $X$-ray, computed tomography scan between $\mathrm{H}$-beam shaped spacer (A-C) and rim-shaped spacer (D-F) (postoperative 12 months).

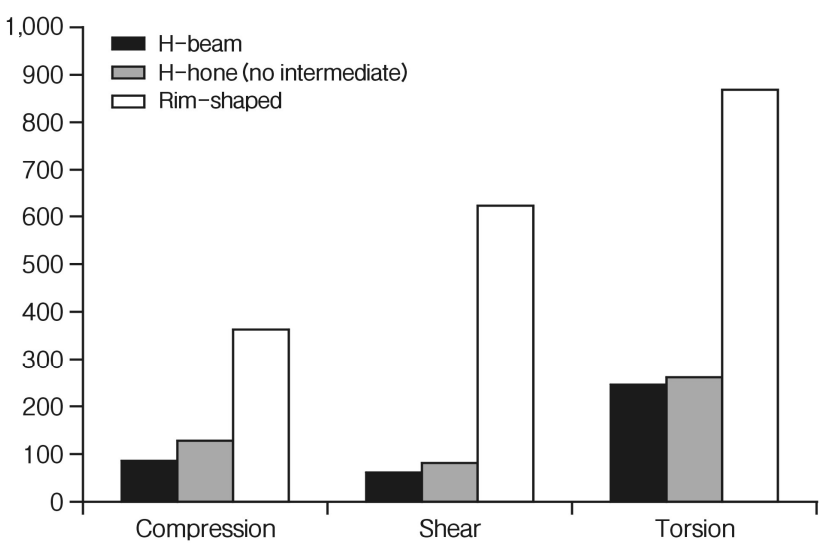

Fig. 3. Finite element method results of peak von Mises stress of compression, shear, and torsion, under the same load.

Table 3. Median absolute and standard deviation improvement in neck and arm pain after treatment in group A and B

\begin{tabular}{lccccccc}
\hline \hline \multirow{2}{*}{ VAS score (pre-post) } & \multicolumn{3}{c}{ Group $A(n=31)$} & & \multicolumn{3}{c}{ Group $B(n=31)$} \\
\cline { 2 - 4 } & 1 l level & 2 level & 3 level & & 1 level & 2 level & 3 level \\
\hline Neck pain & $4.90 \pm 1.20$ & $5.00 \pm 1.15$ & $4.50 \pm 0.71$ & & $5.11 \pm 0.76$ & $5.71 \pm 0.76$ & $5.83 \pm 0.98$ \\
Arm pain & $5.10 \pm 1.37$ & $4.79 \pm 0.54$ & $5.00 \pm 0.00$ & & $5.56 \pm 0.86$ & $5.71 \pm 0.76$ & $5.17 \pm 0.41$ \\
\hline
\end{tabular}

Data are presented as mean \pm standard deviation.

VAS: visual analog scale. 
than that using rim-shaped spacer (Fisher's exact test, $\mathrm{p}>0.05$ ). It is estimated that there are associated with the surface appearance of H-beam spacer. The surface of the H-beam spacer has textured grooves. These grooves can minimize the risk of migration and displacement in anterior-posterior direction. Also, convex superior surface enhances anatomical interface with vertebral endplate-anatomical shape matches vertebral endplate. This shape confirms to anatomy of cervical vertebral bodies and minimizes tissue deformation and damage.

Third, the fusion rate at 12 month follow-ups were no significant difference in fusion rates between H-beam shaped and rim shaped allograft (Fisher's exact test, $\mathrm{p}>0.05$ ). It is considered that there are associated with the hole appearance of H-beam spacer. Large central hole accommodates osteobiologic material packing and promotes fusion through the implant, and the bed of central hole can minimize the risk of migration that it is caused by the force of gravity in inferior direction. Also, $1 \mathrm{~mm}$ pattern holes promote fusion through the implant and help blood circulation.

Forth, the breakage rate among patients who experienced in H-shaped spacer was significantly less likely than that using rim-shaped spacer (Fisher's exact test, $\mathrm{p}<0.05$ ). Single level ACDF surgeries were frequently performed in Group B and multi-level ACDF surgeries (more than 2 levels) were more frequently performed in Group A. Even though 5 patients in Group B showed breakage of allospacer, only 1 patient needed to have additional surgery because of fusion failure. Reinforcement using plate and screw system could give additional stability in spite of the breakage of the allospacer, and so fusion rate between groups was not statistically significant. But, the cases of allospacer breakage showed statistically significant difference (Fisher's exact test, $\mathrm{p}<0.05$ ).

H-beam shaped spacer showed better result than rim shaped spacer in the breakage. We have conducted a FEM study to find the systemic and biomechanical cause for these results. We designed the 3 different shaped spacer models; H-beam shaped, H-hole shaped and rim shaped. Shapes of 3 models were similarly designed to real allospacers which were being used in the current practice. In FEM study, the results were derived from 3 types of spacer that H-beam shaped allobone spacer was the most effectiveness stress distribution. Among the results, under to the compression, shear force load, PVMS values were placed on the thread value. On the other hand, under to the torsion load, H-beam shaped allospacer was showed PVMS value corresponding to $28.3 \%$ relative to other allospacer. It means that the effective stress distribution when the torsion load than the other loads. It is considered that is a great role to apply a support structure (H-shape) in the central part unlike conventional products (rim shaped allospacer). Rim- shaped allospacer was also showed a large PVMS value under the shear, torsion load. This result is thought to be the cause of a thin thickness of rim shaped allospacer. In conclusion, because of many rotary motion of cervical spine, applying a structure in the middle of the spacer is likely to give high stability.

The journals on clinical outcomes and FEM study compared in accordance with the shape of allobone cervical spacers are rare. Perhaps this retrospective study is considered to be the first study comparing the clinical outcome and complication in according to the cervical spacer shape. As mention above, if clinical outcomes and FEM study results is changed in accordance with the cervical spacer shape, we can see that the consideration of the spacer shape is required

However, a limitation of this study is that the 2 groups were not randomly selected for treatment, and thus the groups are not strictly comparable. Another limitation is the small number of patients in each group. Also this study has the limitation of FEM study, not clinical trial.

\section{CONCLUSION}

Cervical allospacer and plate-screw fixation is stable fusion rate with low incidence of complications compared with those of other cervical implants. In ACDF using allograft, H-beam shaped allospacer shows stable fusion rate with low incidence of complication compared with those of other types of allospacer.

\section{CONFLICTS OF INTEREST}

No potential conflict of interest relevant to this article was reported.

\section{REFERENCES}

1. Albert TJ, Murrell SE: Surgical management of cervical radiculopathy. J Am Acad Orthop Surg 7:368-376, 1999

2. An HS, Simpson JM, Glover JM, Stephany J: Comparison between allograft plus demineralized bone matrix versus autograft in anterior cervical fusion. A prospective multicenter study. Spine (Phila Pa 1976) 20:2211-2216, 1995

3. Aronson N, Filtzer DL, Bagan M: Anterior cervical fusion by the smith-robinson approach. J Neurosurg 29:396-404, 1968

4. Baba H, Furusawa N, Imura S, Kawahara N, Tsuchiya H, Tomita $\mathrm{K}$ : Late radiographic findings after anterior cervical fusion for spondylotic myeloradiculopathy. Spine (Phila Pa 1976) 18:21672173, 1993

5. Buttermann GR: Prospective nonrandomized comparison of an allograft with bone morphogenic protein versus an iliac-crest autograft in anterior cervical discectomy and fusion. Spine J 8:426-435, 2008

6. Chesnut RM, Abitbol JJ, Garfin SR: Surgical management of cervical radiculopathy. Indication, techniques, and results. Orthop Clin North Am 23:461-474, 1992

7. Cho DY, Lee WY, Sheu PC: Treatment of multilevel cervical fusion with cages. Surg Neurol 62:378-385, discussion 385-376, 2004 
8. Chou YC, Chen DC, Hsieh WA, Chen WF, Yen PS, Harnod T, et al.: Efficacy of anterior cervical fusion: comparison of titanium cages, polyetheretherketone (PEEK) cages and autogenous bone grafts. J Clin Neurosci 15:1240-1245, 2008

9. Cloward RB: The anterior approach for removal of ruptured cervical disks. 1958. J Neurosurg Spine 6:496-511, 2007

10. Dimitriou R, Mataliotakis GI, Angoules AG, Kanakaris NK, Giannoudis PV: Complications following autologous bone graft harvesting from the iliac crest and using the RIA: a systematic review. Injury 42 Suppl 2:S3-S15, 2011

11. Floyd T, Ohnmeiss D: A meta-analysis of autograft versus allograft in anterior cervical fusion. Eur Spine J 9:398-403, 2000

12. Gercek E, Arlet V, Delisle J, Marchesi D: Subsidence of standalone cervical cages in anterior interbody fusion: warning. Eur Spine J 12:513-516, 2003

13. Kao TH, Wu CH, Chou YC, Chen HT, Chen WH, Tsou HK: Risk factors for subsidence in anterior cervical fusion with standalone polyetheretherketone (PEEK) cages: a review of 82 cases and 182 levels. Arch Orthop Trauma Surg 134:1343-1351, 2014

14. Miller LE, Block JE: Safety and effectiveness of bone allografts in anterior cervical discectomy and fusion surgery. Spine (Phila Pa 1976) 36:2045-2050, 2011

15. Moreland DB, Asch HL, Clabeaux DE, Castiglia GJ, Czajka GA, Lewis PJ, et al.: Anterior cervical discectomy and fusion with implantable titanium cage: initial impressions, patient outcomes and comparison to fusion with allograft. Spine J 4:184-191; discussion 191, 2004

16. Pintar FA, Maiman DJ, Hollowell JP, Yoganandan N, Droese
KW, Reinartz JM, et al.: Fusion rate and biomechanical stiffness of hydroxylapatite versus autogenous bone grafts for anterior discectomy. An in vivo animal study. Spine (Phila Pa 1976) 19: 2524-2528, 1994

17. Pollock R, Alcelik I, Bhatia C, Chuter G, Lingutla K, Budithi C, et al.: Donor site morbidity following iliac crest bone harvesting for cervical fusion: a comparison between minimally invasive and open techniques. Eur Spine J 17:845-852, 2008

18. Samartzis D, Shen FH, Matthews DK, Yoon ST, Goldberg EJ, An HS: Comparison of allograft to autograft in multilevel anterior cervical discectomy and fusion with rigid plate fixation. Spine J 3:451-459, 2003

19. Schneider JR, Bright RW: Anterior cervical fusion using preserved bone allografts. Transplant Proc 8:73-76, 1976

20. Senter HJ, Kortyna R, Kemp WR: Anterior cervical discectomy with hydroxylapatite fusion. Neurosurgery 25:39-42; discussion 42-33, 1989

21. Silber JS, Anderson DG, Daffner SD, Brislin BT, Leland JM, Hilibrand AS, et al.: Donor site morbidity after anterior iliac crest bone harvest for single-level anterior cervical discectomy and fusion. Spine (Phila Pa 1976) 28:134-139, 2003

22. Smith GW, Robinson RA: The treatment of certain cervicalspine disorders by anterior removal of the intervertebral disc and interbody fusion. J Bone Joint Surg Am 40-a:607-624, 1958

23. Yue WM, Brodner W, Highland TR: Long-term results after anterior cervical discectomy and fusion with allograft and plating: a 5- to 11-year radiologic and clinical follow-up study. Spine (Phila Pa 1976) 30:2138-2144, 2005 